\title{
Evaluation of medical quality and treatment trends of nonvalvular atrial fibrillation in Beijing inpatients
}

\author{
Hai-Rong Yu ${ }^{1,2}$, Chang-Sheng $\mathrm{Ma}^{1}$, Xin $\mathrm{Du}^{1}$, San-Shuai Chang ${ }^{1}$, Jian-Zeng Dong ${ }^{1}$ \\ ${ }^{1}$ Department of Cardiology, Beijing Anzhen Hospital, Capital Medical University, National Clinical Research Center for Cardiovascular Diseases, \\ Beijing Advanced Innovation Center for Big Data-Based Precision Medicine for Cardiovascular Diseases, Beijing, China; ${ }^{2}$ Cardiovascular Center, \\ Beijing Tongren Hospital, Capital Medical University, Beijing, China \\ Contributions: (I) Conception and design: HR Yu; (II) Administrative support: JZ Dong; (III) Provision of study materials or patients: HR Yu; (IV) \\ Collection and assembly of data: CS Ma, X Du; (V) Data analysis and interpretation: SS Chang, JZ Dong; (VI) Manuscript writing: All authors; (VII) \\ Final approval of manuscript: All authors. \\ Correspondence to: Dr. Jian-Zeng Dong. Department of Cardiology, Beijing Anzhen Hospital, Capital Medical University, National Clinical Research \\ Center for Cardiovascular Diseases, Beijing Advanced Innovation Center for Big Data-Based Precision Medicine for Cardiovascular Diseases, No. 2, \\ Anzhen Rd, Chaoyang District, Beijing 100029, China. Email: jz_dong@126.com.
}

Background: The quality of care presented to AF cases in tertiary and non-tertiary hospitals in China remains obscure and needs further investigation. Therefore, we examined the compliance with quality measures (QMs) and clinical performance measures (PMs) that were proposed by the ACC/AHA in 2016 in a Chinese adult population with nonvalvular atrial fibrillation (AF). We also investigated the changes in patients' characteristics, treatment plans, and quality of provided care at different time-points [2011-2016].

Methods: We used the datasets from two registry-based studies in Beijing, including the Chinese Atrial Fibrillation Registry (CHINA-AF) investigation. From August 2011 to July 2016, an overall number of 13,439 nonvalvular AF cases were included. Our primary outcome was to determine the compliance rate with two PMs [oral anticoagulant (OAC) use and prothrombin time international normalized ratio (INR)] and six QMs (beta-blocker, ACEI-ARB, and the inappropriate prescription of antiarrhythmic drugs, Sotalol, antiplatelet and OAC, and non-dihydropyridine calcium channel antagonist) over a period of 5 years [20112016]. Also, the difference in compliance between tertiary and non-tertiary hospitals was investigated.

Results: The compliance with PMs and QMs was variable between tertiary and non-tertiary hospitals. In tertiary hospitals, the total number of inpatient beds was $>500$, and each bed was equipped with $>1.03$ health technical personnel and $>0.4$ nurses. In non-tertiary hospitals, the available beds capacity was $<500$, and each bed corresponded with $0.7-0.88$ health technical personnel and $>0.4$ nurses. The compliance rates with OAC use steadily increased from 2011-2015 in tertiary hospitals (35\%, 35.9\%, 37.9\%, 45\%, and 49\%, $\mathrm{P}<0.001)$. In non-tertiary hospitals, INR follow-up of warfarin use increased significantly over the years, with rates of $36.2 \%, 60.5 \%, 71.6 \%, 64.3 \%$, and $81.5 \%(\mathrm{P}=0.04)$, respectively. In terms of QMs, the compliance with the six measure sets was quite similar among tertiary and non-tertiary hospitals avoiding any significant difference over the years $(\mathrm{P}>0.05)$.

Conclusions: We noted an improvement in medical quality regarding the use of OAC, especially in tertiary hospitals. Meanwhile, the compliance rates of INR follow-up improved in non-tertiary hospitals. However, the compliance rates of other performance and QMs are still low, which are in need of improvement.

Keywords: Atrial fibrillation (AF); performance measure (PM); quality measure (QM); compliance

Submitted Sep 26, 2020. Accepted for publication Apr 08, 2021.

doi: 10.21037/apm-20-1925

View this article at: http://dx.doi.org/10.21037/apm-20-1925 


\section{Introduction}

The occurrence of atrial fibrillation (AF) is increasing continuously with an enhanced risk of heart failure (HF), stroke, and death (1-3). It is linked with impaired quality of life (QOL), along with an enhanced risk of overall mortality for almost $40-90 \%$ (4). The incidence of AF in China ranges from $0.77 \%$ to $1.03 \%$ (5), and according to age, up to 4.2 million patients ( $\geq 30$ years) are diagnosed with $\mathrm{AF}$ in China (5).

Based on the mortality, morbidity, and costs correlated with atrial flutter (At-F) and AF, the American College of Cardiology (ACC), American Heart Association (AHA), and Physician Consortium highlighted the importance of performance measures (PMs) in raising the level of awareness among healthcare providers, as well as in implementing these guidelines in clinical practice. With the help of these tools, the physicians can perform a better assessment regarding the quality of provided care among nonvalvular $\mathrm{AF}$ and At-F patients (6).

In the past, PMs related to the treatment and diagnosis of AF and atrial flutter were released in 2008 (6), and they were later implemented in 2011 (7). Back then, PMs included only 3 items: thromboembolic risk factors assessment, therapy of chronic anticoagulation, and the measurement of international normalized ratio (INR) on a monthly basis. These PMs are applied in patients of nonvalvular AF or At-F which was not caused by acute reversible events (6). In 2016, the ACC/AHA clinical guidelines distinguished quality measures (QMs) from PMs, with a total of 24 measure sets. These measures included 6 PMs (3 in-patient and 3 out-patient measures) and 18 QMs (10 in-patient and 8 out-patient measures) (8).

Although clinical practice guidelines that are based on recent evidence improved patients' outcomes (9-11), the risk of AF has not been effectively controlled. Some studies reported a very low compliance rate with evidence-based therapies, including the assessment of thromboembolic risk, anticoagulation therapy, and adequate treatment of concomitant diseases $(12,13)$. Meanwhile, the cost of care for patients with $\mathrm{AF}$ is high (14).

Previous registry-based studies assessed the use of anticoagulant therapy (15) and evidence-based statins for the treatment of AF (16). However, according to treatment guidelines proposed via the European Society of Cardiology (ESC) in 2016, AF requires a comprehensive management plan, including the management and detection of risk factors and cardiovascular diseases appearing at the same time, prevention of stroke, and the control of heart rate or rhythm (11). There is a persuasive need regarding the systematic measurement of the quality of care in AF patients, as quantified by established measures for the identification of potential gaps and providing opportunities regarding quality improvement.

Despite the fact that our comprehension of the clinical practice of AF in China has improved due to the increased number of registry-based studies, these reports are limited in the representativeness of hospitals, and they lack the assessment towards the improvement of different quality components. Therefore, we conducted the current investigation to assess trends in characteristics, treatment, and quality of care provided to nonvalvular AF patients. For these purposes, we analyzed the data from two studies, including the China Atrial Fibrillation Registry (CHINAAF) study (17) and the study of Chang et al. (15), based on the 2016 ACC/AHA clinical performance and QMs in AF or At-F adults.

We present the following article in accordance with the MDAR checklist (available at http://dx.doi.org/10.21037/ apm-20-1925).

\section{Methods}

CAFR is a multicenter, prospective, hospital-based registry of nonvalvular AF cases (ChiCTR-OCH-13003729). The CHINA-AF study was first launched in August 2011 with the intention to recruit approximately 20,000 AF patients over several years while following-up these patients every six months (17). The primary outcome of this observation was to investigate the current trend of the clinical practice in Beijing and compare various $\mathrm{AF}$ treatment plans occurring in real-world practices. A total of 13,439 AF patients in 12 non-tertiary and 20 tertiary hospitals, site for the treatment of a majority of patients in Beijing, were included. On the other hand, the study of Chang et al. (15) involved hospitalized patients with $\mathrm{AF}$ in Beijing who were not included in the CHINA-AF study. Participating hospitals in the mentioned studies were mostly in Beijing (tertiary and non-tertiary), providing clinical care to AF patients. Tertiary hospitals fall in level 3 hospitals (major centers with in-patients bed of $\geq 500$, providing high-quality medical facilities to several regions). On the other hand, non-tertiary hospitals are comprised of both level 1 (basic facilities bearing community hospitals) and level 2 hospitals which possess $\geq 100$ in-patient beds serving a population of $\geq 100,000$. Non-tertiary hospitals 
included primary hospitals and secondary hospitals. Firstclass hospitals are health centers and primary hospitals that directly provide service to the communities in terms of treatment, prevention and health care, and rehabilitation services. Secondary hospitals describe a set of hospitals that meet the Chinese hospital-grade standards. The comprehensive medical and health services are provided by various regional hospitals dealing with multiple communities and work on certain teaching and scientific research. County, district, and municipal hospitals are all above level 2. A detailed description of China hospitals (non-tertiary and tertiary) is provided in (Table S1).

We obtained treatment data of included patients from medical records. The collected data were mainly electronic and possess defined broadness and acceptability checks provided by the respective projects of quality assurance. All data were retrieved from the Chinese Atrial Fibrillation Registry (CHINA-AF) research, a real-world registry research conducted in Beijing, China. The aim of the CHINA-AF research is to provide evidence of real-world data based clinical practice. Of the 33 tertiary and 49 nontertiary hospitals treating AF patients in Beijing, 32 tertiary and non-tertiary hospitals also agree to participate in this study.

\section{Study population}

The population of study was based on the data reported in the afore mentioned two registry-based studies. The review and approval of both studies were acquired from the Ethics Committee of Beijing Anzhen Hospital (D11110700300000). The study conformed to the provisions of the Declaration of Helsinki (as revised in 2013). Informed consent was obtained from all participants. Cases $\geq 18$ years of age, with six months AF documentation provided either by ECG or Holter were eligible for inclusion. Meanwhile, transient and reversible AF patients which was caused by specific precipitating or reversible causes (hyperthyroidism, cardiothoracic surgery, myocardial infarction, pericarditis, myocarditis, pulmonary embolism, electrocution, and binge drinking), patients with other and life expectancy of less than one year, rheumatic mitral stenosis was diagnosed in patients, and those with mitral valve prostheses were excluded.

Based on our inclusion criteria, all eligible cases with a diagnosis of AF were encouraged to participate in this study. Written consent was provided from each eligible patient prior to participation. Trained research coordinators and cardiologists interviewed the patients, and abstracted relevant data from their medical records. These data included demographic characteristics, medical history, and treatment plans. Into a system of web-based data capture, all data are recorded.

For analysis purposes, recruited patients were distributed in five groups based on discharge time (2011.8.1-2012.7.31, 2012.8.1-2013.7.31, 2013.8.1-2014.7.31, 2014.8.12015.7.31, and 2015.8.1-2016.7.31) in order to assess the changes in compliance rates over the years. Baselines characteristics of included participants based on the mentioned time-points are summarized in Table 1.

\section{Quality indicators}

The construction of investigated quality indicators was based on the clinical behavior of 2016 ACC/AHA and QMs for AF or At-F inpatient adults (8). In our study, the analysis of inpatients with AF included two PMs and six QMs. The two PMs were: (I) prescription of anticoagulation prior to discharge and (II) follow-up documentation of PT-INR plan before the discharge for warfarin treatment.

Meanwhile, the six QMs were: (I) before discharge a prescription of Beta-blocker [when left ventricular ejection fraction (LVEF) $\leq 40$ ]; (II) Angiotensin-Converting Enzyme Inhibitor-Angiotensin Receptor Blocker (ACEI$\mathrm{ARB}$ ) prescribed before discharge (when LVEF $\leq 40$ ); (III) improper prescription of antiarrhythmic medications before discharge to cases with permanent AF for the control of rhythm; (IV) improper prescription of Sotalol before discharge in cases with $\mathrm{AF}$ and end-stage kidney malady or on dialysis before discharge; $(\mathrm{V})$ improper prescription of oral anticoagulation and antiplatelet therapy before discharge for cases without coronary artery disease (CAD) and/or vascular disease; and (VI) improper prescription of non-dihydropyridine calcium channel antagonist before discharge in cases with decreased EF HF (when LVEF $\leq 40$ ).

Noteworthy, the score of $\mathrm{CHA}_{2} \mathrm{DS}_{2}$-VASc, a PM, was examined for each case by the assignment of 1 point for each factor present. These factors included age of 65-74 years, hypertension history, diabetes mellitus $(\mathrm{DM})$, vascular disease (CAD or peripheral artery disease), congestive cardiac failure, and female sex. Meanwhile, 2 points were given in two conditions: (I) age $\geq 75$ years and (II) historical background of stroke/ TIA/thromboembolism (18). High-risk AF patients were defined if they had a risk score of $\geq 2$. Other performance and QMs could not be adequately assessed along with 


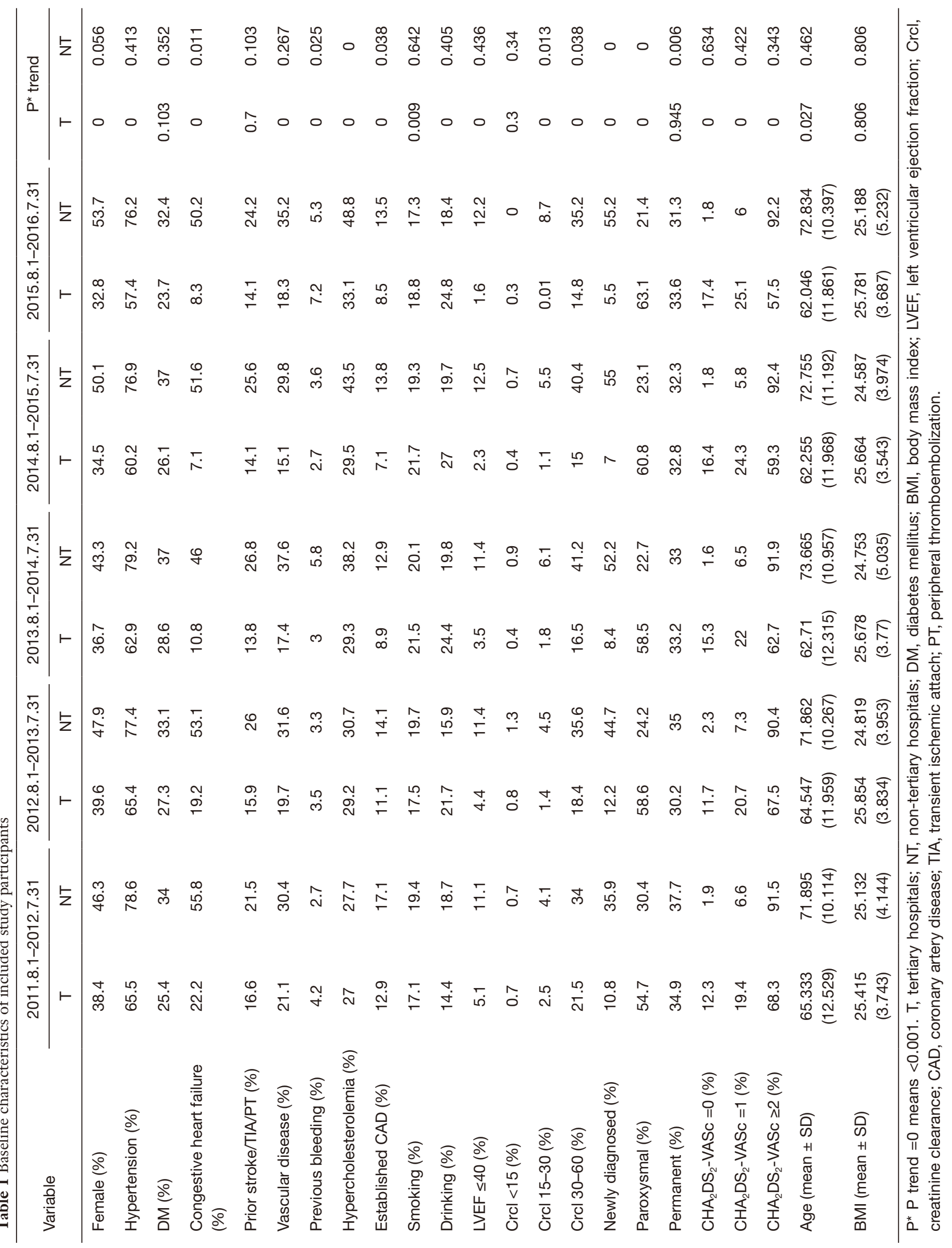


their compliance rates due to the lack of relevant data.

\section{Research results}

The main objective of the present research was the assessment of the rate of compliances with each of the aforementioned performances of ACC/AHA and QMs among inpatients with AF. The compliance rate of each measure was described as the numbers of cases who met that certain measure separated through the overall number of eligible cases. The numerator includes the number of patients who met the PMs and QMs, while the denominator includes the overall number of patients eligible for PMs and QMs. Patients were found to be eligible if they fulfill the eligibility criteria of PMs and did not have a medical (i.e., use of beta-blocker in hypotension or bradycardia patients) or personal (i.e., refusal of patients to take warfarin for $\mathrm{AF}$ ) contraindication for that measure (19).

\section{Statistical analysis}

Data of baseline demographic characteristics and medical history were indicated as means \pm standard deviations (SD) for continuous variables, while numbers and proportions were given for categorical variables. We examined the characteristics of patients, tests, procedures, treatments, and crude rate of compliance across different study years. As regards trend analysis, we used the test of MannKendall trend for continuous variables and the Cochran Armitage trend assessment for binary variables. To examine the temporal changes, we defined a time variable "Ty". Based on the enrollment time, patients were divided into five groups. Patients enrolled from (August 1, 2011) to (July 31, 2012) were denoted as " 0 ", those enrolled from (August 1, 2012) to (July 31, 2013) were named as "1", those enrolled from (August 1, 2013) to (July 31, 2014) were assigned as "2", those enrolled from (August $1,2015)$ to (July 31,2015 ) were mentioned as " 3 ", and those enrolled from (August 1, 2015) to (July 31, 2016) were represented as " 4 ". Then, a logistic regression model was established, with performance evaluation or quality evaluation as the dependent variable. We investigated the corresponding $\mathrm{P}$ value of Ty while adjusting for the baseline characteristics. All comparison of this study was two-sided, with statistical significance described as a $\mathrm{P}$ value of less than 0.05 .

Missing data in our study were considered missing completely at random (MCAR) since the data were missing regardless of the exposure or outcome status of included patients. The registry that we used in our study report data of patients with AF independent of the outcome in these patients. Therefore, we assumed that no systematic differences exist between those with missing and those with complete records. In this regard, we used the complete case analysis method, where patients with complete records for all outcome variables were incorporated in the planned analysis. Meanwhile, cases were excluded from analysis in case of any missing data on any variable. All statistical investigations were executed through SAS software (version 9.2, SAS Institute, Cary, $\mathrm{NC}$ ) and R software (version 3.0.2, R Foundation for Statistical Computing, Vienna, Austria).

\section{Results}

\section{Patient characteristics}

An overall number of 13,439 cases were enrolled in the study, of whom 986 were recruited from tertiary hospitals, and 3,573 were enrolled from non-tertiary hospitals. Table 1 comprises the baseline features.

The proportion of cases with a history of stroke, congestive cardiac failure, hypertension vascular disease, hypercholesterolemia, CAD, smoking, and drinking as well as the proportion of patients with LVEF ( $<40 \%)$, creatinine clearance (15:60), newly diagnosed AF, and CHA2DS2-VASc $\geq 2$ showed a significant decrease in tertiary hospitals than non-tertiary ones $(\mathrm{P}<0.05)$. Moreover, the age and number of female patients were significantly less in non-tertiary hospitals $(\mathrm{P}<0.05)$. While, the percentage of patients with a previous bleeding disorder, paroxysmal AF, and CHA2DS2-VASc (0 and 1) increased significantly among tertiary hospitals compared to non-tertiary ones $(\mathrm{P}<0.05)$.

\section{PMs}

Two PMs were analyzed in our study. Patients who underwent AF ablation within three months from anticoagulant therapy were removed from the calculation of the anticoagulation ratio. In tertiary hospitals, the proportions of nonvalvular AF patients (CHA2DS2-VASc score $\geq 2$ ) receiving oral anticoagulants (OAC) [novel oral anticoagulants (NOACs) and warfarin] before discharge (from 2011 to 2015 ) were $35 \%, 35.9 \%, 37.9 \%$, 45\%, and $49 \%$ respectively $(\mathrm{P}<0.001)$ (Figure 1$)$. Meanwhile, 
PM and QM of inpatient in tertiary hospitals

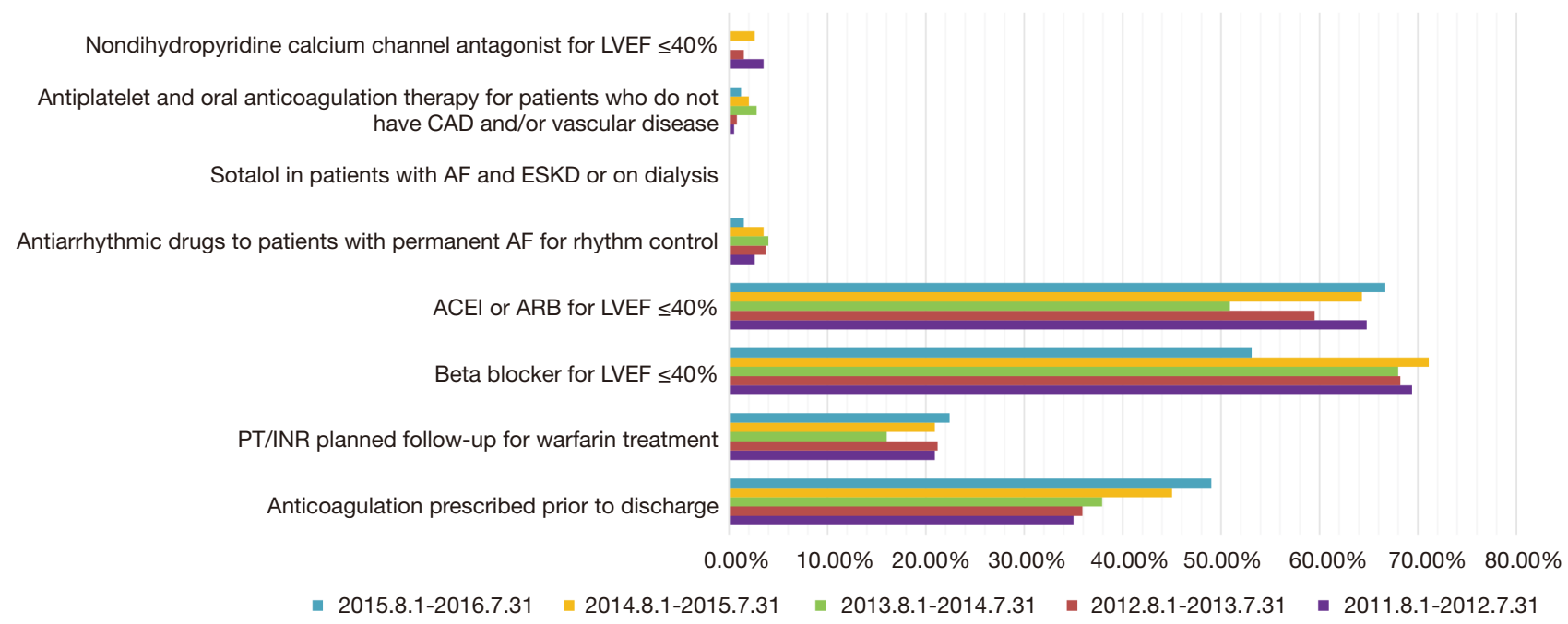

PM: Clinical Performance; QM: Quality Measures; LVEF: Left Ventricular Ejection Fraction; CAD: Coronary Artery Disease; AF: Atrial Fibrillation; ESKD: End-Stage Kidney Disease; ACEI: Angiotensin-Converting Enzyme Inhibitor; ARB: Angiotensin Receptor Blocker; PT: Peripheral Thromboembolization; INR: International Normalized Ratio.

Figure 1 Compliance rates of PM and QM in tertiary hospitals.

the proportions of patients who received OAC prior to discharge in non-tertiary hospitals were $9.2 \%, 11.2 \%$, $10.8 \%, 10.8 \%$, and $11.9 \%$, respectively $(\mathrm{P}=0.214)$ (Figure 2$)$.

Furthermore, the proportion of patients who had INR follow-up plan for warfarin treatment in non-tertiary hospitals were $36.2 \%, 60.5 \%, 71.6 \%, 64.3 \%$, and $81.5 \%$, respectively $(\mathrm{P}=0.04)$, while the compliance rates in tertiary hospitals were $20.9 \%, 21.2 \%, 16 \%, 20.9 \%$, and $22.4 \%$, respectively $(\mathrm{P}=0.891)$ (Figures 1,2$)$.

\section{QMs}

The compliance rates with six QMs were reported among tertiary and non-tertiary hospitals (Figures 1,2). As AF radiofrequency ablation cannot be implemented in nontertiary hospitals, the assessment of AF catheter ablation in patients with or without postoperative anticoagulation could not be performed.

Except for QM-5 (improper prescription of antiplatelet and OAC therapy before discharge for cases who do not have the vascular disease/or coronary artery disease), all of the six QMs revealed no significant increase in the patients using antiplatelet and OAC drugs before discharge in nontertiary and tertiary hospitals. Meanwhile, as regard QM-5, the proportion of patients differed significantly in tertiary $(\mathrm{P}=0.037)$ and non-tertiary hospitals $(\mathrm{P}=0.027)$.

\section{Discussion}

This is the first descriptive assessment of the 2016 ACC/ AHA PMs and QMs for AF in cases in China. In this study, we provide contemporary information on the medical quality and treatment trends of inpatients with nonvalvular $\mathrm{AF}$ among a large set of Chinese patients in clinical practice. Our study highlights two major findings. Firstly, we noted that the proportion of nonvalvular AF patients (CHA2DS2VASc score $\geq 2$ ), in our study, who received OAC (warfarin and NOACs) prior to discharge increased significantly over the years (five time-points) in tertiary hospitals with rates of $35 \%, 35.9 \%$, 37.9\%, 45\%, and 49\%, respectively. However, our finding is inconsistent with the reported rates of $80 \%$ and $85.6 \%$ in the United States (U.S.) (from the National Cardiovascular Data Registry's Practice Innovation and Clinical Excellence (PINNACLE) registry in 2010) (19) and Japan (from the Japanese Multicenter Registry) (20), respectively. Secondly, in our study, the proportion of INR follow-up plans for warfarin treatment in non-tertiary hospitals increased significantly over the years with rates of $36.2 \%, 60.5 \%, 71.6 \%, 64.3 \%$, and $81.5 \%$, respectively. However, these rates are still lower than the reported rate in Japan of $90.3 \%$ (20).

Meanwhile, the quality of medical care provided for AF patients remains the decisive factor in determining the 
PM and QM of inpatient in nontertiary hospitals

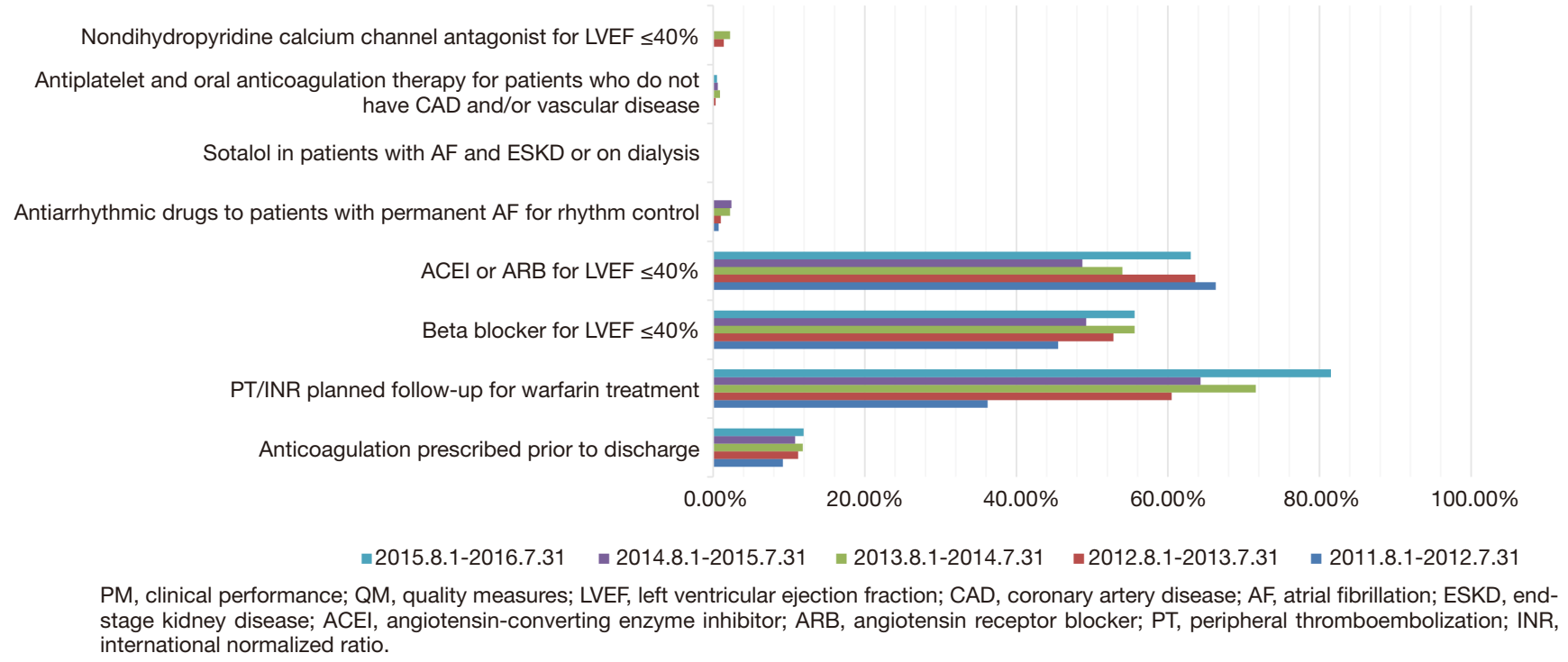

Figure 2 Compliance rates of PM and QM in non-tertiary hospitals.

clinical prognosis of such patients. However, the medical quality provided for patients with $\mathrm{AF}$ in China is not well understood. The core evaluation index for the treatment of AF is the rate of anticoagulant use. Previous studies reported that low rates of $\mathrm{AF}$ patients in China are treated with anticoagulant therapy (21).

This study highlights the significant increase in the rate of anticoagulant treatment usage in tertiary hospitals in Beijing over the past 5 years. On the other hand, there was no obvious change in the rate of anticoagulant treatment in non-tertiary hospitals. This is inseparable from the continuous learning guidelines for primary healthcare physicians, along with the application of these guidelines in clinical practice at tertiary hospitals. Compared with the non-tertiary hospital doctors, doctors in tertiary hospitals have more opportunities to study and apply the acquired experience in clinical practice, and thus, the application of anticoagulant treatment in patients with AF has greatly improved. Secondary to the current situation of the country, a lot of tertiary hospitals in Beijing have set up specialized outpatient clinics for AF. Many patients with AF primarily address tertiary hospitals. Therefore, doctors in those hospitals have more patients with $\mathrm{AF}$, more clinical exposure, higher experience, and more importantly, more insight into the optimum anticoagulant treatment of such patients.

The use of OAC is closely related to the economic level, the promotion of public health literacy, and the training of doctors. Our study showed that the rates of anticoagulant use from 2011 to 2013 increased from $35 \%$ to $35.9 \%$ to $37.9 \%$, respectively. Meanwhile, in 2014, a more significant pronounced increase was noted with a rate of $45 \%$. This could be related to the approval of Dabigatran use in China in May 2013. In the same context, in 2015, the rates of anticoagulant use reached $49 \%$. This could be related to the approval of Rivaroxaban use in China as an anticoagulant drug for nonvalvular AF patients with one or more risk factors.

Compared with developed countries, the rate of use of anticoagulant therapy in high-risk AF patients remains very low in China. GARFIELD-AF registration study reported that $62 \%$ of high-risk patients with AF (CHA2DS2-VASc 22 ) received OAC therapy (22). Such a low rate in our population could be explained by various factors, including the increased number of cases with AF in our country, the presence of no sufficient communication between doctors and patients, patients concerns about the risk of bleeding, and the high cost of anticoagulant treatment (15).

Noteworthy, compared with tertiary hospitals, the compliance rate with INR follow-up plans during warfarin treatment in non-tertiary hospitals has increased significantly over the years from 2011 to 2015 . This indicates that doctors in non-tertiary hospitals pay significant attention to the quality and follow-up of 
anticoagulant treatment in At-F patients. Tertiary hospitals revealed no considerable change in the compliance rate of INR follow-up in cases with AF over the years. This may be due to the less number of patients in non-tertiary hospitals compared with tertiary hospitals, and thus, doctors have sufficient time to communicate and discuss the treatment plan thoroughly with the patients. At the same time, nontertiary hospitals are more adjacent to larger communities. Therefore, it is more convenient for patients with AF to go to non-tertiary hospitals to follow-up INR during anticoagulant therapy.

After taking warfarin, INR needs to be tested in the hospital. With the developing economy of China, many non-tertiary hospitals have had facilities for running INR testing. The doctors in the tertiary hospitals are unable to provide detailed, thorough follow-up and monitoring plans for patients due to the increased number of presenting patients. Presently, Beijing city gradually establishes a hierarchical treatment pattern for AF patients. A significant proportion of AF patients in tertiary hospitals, who have completed the assessment of AF treatment, will go to the medical body cooperation hospital, a non-tertiary hospital, for follow-up in the long-term. It is also essential that healthcare providers at tertiary hospitals follow-up their patients' conditions in the long-term for a better quality of care.

Noteworthy, as regards QMs, the compliance rate with the analyzed QM sets remained low and does not significantly increase over the years. Moreover, their compliance rates were quite similar between tertiary and non-tertiary hospitals. As for the QMs of the utilization of beta-blockers and ACEI/ARBs in cases with LVEF of $\leq 40 \%$, the compliance rate with these measures was comparable to that reported in western countries, while in Japan, the compliance rate with the use of ACEI and betablockers was $35 \%$ and $54 \%$ (20).

Our study had several limitations in sample selection, quality of data collected from medical records, and confounding factors adjustment. Firstly, all of the participating centers in the analyzed registries are located in Beijing, the capital of China, which is not representative of the whole population. Secondly, we could not analyze the compliance rates of $\mathrm{CHA}_{2} \mathrm{DS}_{2}$-VASc score, which is a very important $\mathrm{PM}$ of $\mathrm{AF}$ due to the lack of related data in our registry records. Thirdly, many potential confounding variables could not be controlled due to the lack of data. Lastly, NOACs were not available until 2013 in China, and these drugs were not supported by medical insurance until
2018. This could have affected the use of OAC drugs in our study due to cost-related issues. Throughout the study, all data were collected from telephone inquiries or outpatient interviews. Due to the difference in the educational level of each patient, there may be a deviation in understanding the problem, which can also have a certain impact on the study.

In conclusion, our study provides an insight into the compliance rate with the clinical performance and QMs proposed by the $2016 \mathrm{ACC} / \mathrm{AHA}$ protocols for the treatment of inpatients with $\mathrm{AF}$. The compliance rate with the use of OAC therapy in tertiary hospitals increased significantly from 2011 to 2015 , with negligible change in non-tertiary hospitals. Meanwhile, the compliance with INR follow-up in patients taking warfarin was shown to have a pronounced increase from $36.2 \%$ to $81.5 \%$ from 2011 to 2015. As for the majority of QMs, no significant change in compliance rates was noted in both non-tertiary and tertiary hospitals over the decades.

\section{Acknowledgments}

Funding: This study was supported by the National Key Research and Development Program of China (2016YFC1301002, 2016YFC0900901, 2017YFC0908803, 2018YFC1312501), and Beijing Municipal Commission of Science and Technology (D171100006817001).

\section{Footnote}

Reporting Checklist: The authors have completed the MDAR reporting checklist. Available at http://dx.doi.org/10.21037/ apm-20-1925

Data Sharing Statement: Available at http://dx.doi. org/10.21037/apm-20-1925

Conflicts of Interest: All authors have completed the ICMJE uniform disclosure form (available at http://dx.doi. org/10.21037/apm-20-1925). The authors have no conflicts of interest to declare.

Etbical Statement: The authors are accountable for all aspects of the work in ensuring that questions related to the accuracy or integrity of any part of the work are appropriately investigated and resolved. Both studies were surveyed and confirmed through the Ethics Committee of Beijing Anzhen Hospital (D11110700300000). The study conformed to the provisions of the Declaration of Helsinki 
(as revised in 2013), and informed consent was obtained from all participants.

Open Access Statement: This is an Open Access article distributed in accordance with the Creative Commons Attribution-NonCommercial-NoDerivs 4.0 International License (CC BY-NC-ND 4.0), which permits the noncommercial replication and distribution of the article with the strict proviso that no changes or edits are made and the original work is properly cited (including links to both the formal publication through the relevant DOI and the license). See: https://creativecommons.org/licenses/by-nc-nd/4.0/.

\section{References}

1. Benjamin EJ, Chen PS, Bild DE, et al. Prevention of atrial fibrillation: report from a national heart, lung, and blood institute workshop. Circulation 2009;119:606-18.

2. Fuster V, Ryden LE, Cannom DS, et al. ACC/AHA/ESC 2006 guidelines for the management of patients with atrial fibrillation: full text: a report of the American College of Cardiology/American Heart Association Task Force on practice guidelines and the European Society of Cardiology Committee for Practice Guidelines (Writing Committee to Revise the 2001 guidelines for the management of patients with atrial fibrillation) developed in collaboration with the European Heart Rhythm Association and the Heart Rhythm Society. Europace 2006;8:651-745.

3. Schnabel RB, Sullivan LM, Levy D, et al. Development of a risk score for atrial fibrillation (Framingham Heart Study): a community-based cohort study. Lancet 2009;373:739-45.

4. Benjamin EJ, Wolf PA, D'Agostino RB, et al. Impact of atrial fibrillation on the risk of death: the Framingham Heart Study. Circulation 1998;98:946-52.

5. Li Y, Wu YF, Chen KP, et al. Prevalence of atrial fibrillation in China and its risk factors. Biomed Environ Sci 2013;26:709-16.

6. Estes NA, 3rd, Halperin JL, Calkins H, et al. ACC/ AHA/Physician Consortium 2008 Clinical Performance Measures for Adults with Nonvalvular Atrial Fibrillation or Atrial Flutter: a report of the American College of Cardiology/American Heart Association Task Force on Performance Measures and the Physician Consortium for Performance Improvement (Writing Committee to Develop Clinical Performance Measures for Atrial Fibrillation) Developed in Collaboration with the Heart Rhythm Society. J Am Coll Cardiol 2008;51:865-84.
7. Peterson E, Albert N, Heidenreich P. Implementation notes 1.0 for the ACC/AHA/Physician Consortium 2008 Clinical Performance Measures for Adults with Nonvalvular Atrial Fibrillation or Atrial Flutter. American College of Cardiology Foundation, American Heart Association, and American Medical Association, 2012. 2008.

8. Heidenreich PA, Solis P, Estes NAM 3rd, et al. 2016 ACC/ AHA Clinical Performance and Quality Measures for Adults With Atrial Fibrillation or Atrial Flutter: A Report of the American College of Cardiology/American Heart Association Task Force on Performance Measures. J Am Coll Cardiol 2016;68:525-68.

9. Camm AJ, Kirchhof P, Lip GY, et al. Guidelines for the management of atrial fibrillation: the Task Force for the Management of Atrial Fibrillation of the European Society of Cardiology (ESC). Europace 2010;12:1360-420. Erratum in: Europace 2011;13:1058.

10. January CT, Wann LS, Alpert JS, et al. 2014 AHA/ACC/ HRS guideline for the management of patients with atrial fibrillation: executive summary: a report of the American College of Cardiology/American Heart Association Task Force on practice guidelines and the Heart Rhythm Society. Circulation 2014;130:2071-104.

11. Kirchhof P, Benussi S, Kotecha D, et al. 2016 ESC Guidelines for the management of atrial fibrillation developed in collaboration with EACTS. Eur J Cardiothorac Surg 2016;50:e1-e88.

12. Zhang H, Yang Y, Zhu J, et al. Baseline characteristics and management of patients with atrial fibrillation/ flutter in the emergency department: results of a prospective, multicentre registry in China. Intern Med J 2014;44:742-8.

13. Zhang S. Atrial fibrillation in mainland China: epidemiology and current management. Heart 2009;95:1052-5.

14. Mozaffarian D, Benjamin EJ, Go AS, et al. Heart disease and stroke statistics--2015 update: a report from the American Heart Association. Circulation 2015;131:e29-322.

15. Chang SS, Dong JZ, Ma CS, et al. Current Status and Time Trends of Oral Anticoagulation Use Among Chinese Patients With Nonvalvular Atrial Fibrillation: The Chinese Atrial Fibrillation Registry Study. Stroke 2016;47:1803-10.

16. Xia SJ, Du X, Li C, et al. Uptake of evidence-based statin therapy among atrial fibrillation patients in China: A report from the CAFR (Chinese Atrial Fibrillation Registry) Study. Int J Cardiol 2016;220:284-9. 
17. Du X, Ma C, Wu J, et al. Rationale and design of the Chinese Atrial Fibrillation Registry study. BMC Cardiovasc Disord 2016;16:130.

18. Lip GY, Nieuwlaat R, Pisters R, et al. Refining clinical risk stratification for predicting stroke and thromboembolism in atrial fibrillation using a novel risk factor-based approach: the euro heart survey on atrial fibrillation. Chest 2010;137:263-72.

19. Chan PS, Oetgen WJ, Buchanan D, et al. Cardiac performance measure compliance in outpatients: the American College of Cardiology and National Cardiovascular Data Registry's PINNACLE (Practice Innovation And Clinical Excellence) program. J Am Coll Cardiol 2010;56:8-14.

20. Inohara T, Kimura T, Ueda I, et al. Effect of Compliance

Cite this article as: Yu HR, Ma CS, Du X, Chang SS, Dong JZ. Evaluation of medical quality and treatment trends of nonvalvular atrial fibrillation in Beijing inpatients. Ann Palliat Med 2021;10(5):5270-5279. doi: 10.21037/apm-20-1925 to Updated AHA/ACC Performance and Quality Measures Among Patients With Atrial Fibrillation on Outcome (from Japanese Multicenter Registry). Am J Cardiol 2017;120:595-600.

21. Steinberg BA, Gao H, Shrader P, et al. International trends in clinical characteristics and oral anticoagulation treatment for patients with atrial fibrillation: Results from the GARFIELD-AF, ORBIT-AF I, and ORBIT-AF II registries. Am Heart J 2017;194:132-40.

22. Oldgren J, Healey JS, Ezekowitz M, et al. Variations in cause and management of atrial fibrillation in a prospective registry of 15,400 emergency department patients in 46 countries: the RE-LY Atrial Fibrillation Registry. Circulation 2014;129:1568-76. 
Table S1 The difference between tertiary and non-tertiary hospitals

\begin{tabular}{|c|c|c|}
\hline & Tertiary hospitals & Non-tertiary hospitals \\
\hline $\begin{array}{l}\text { Department } \\
\text { setting }\end{array}$ & $\begin{array}{l}\text { Emergency room, internal medicine, surgery, gynecology } \\
\text { (obstetrics), preventive health care department, pediatrics, } \\
\text { ophthalmology, otolaryngology, Stomatology, dermatology, } \\
\text { infectious diseases, traditional Chinese medicine, } \\
\text { rehabilitation and medical technology departments at } \\
\text { least have pharmacy, laboratory, Radiology, operating } \\
\text { room, pathology, nuclear medicine, blood transfusion, } \\
\text { physiotherapy (can be combined with rehabilitation), } \\
\text { disinfection and supply room, medical record room, } \\
\text { nutrition department and medical technology department } \\
\text { Corresponding clinical function examination room }\end{array}$ & $\begin{array}{l}\text { Otolaryngology, Stomatology, Dermatology and infectious } \\
\text { diseases, among which department of Ophthalmology, } \\
\text { Department of Otolaryngology and Department of } \\
\text { Stomatology can be combined, Department of Dermatology } \\
\text { can be combined with Department of internal medicine or } \\
\text { surgery. The medical technology department at least has } \\
\text { pharmacy department, laboratory, radiology department, } \\
\text { physiotherapy department, disinfection and supply room, } \\
\text { operation room, pathology room, blood bank (which } \\
\text { can be incorporated into the laboratory and equipment), } \\
\text { physiotherapy room and medical record room }\end{array}$ \\
\hline
\end{tabular}

Facilities and Each bed shall be equipped with at least 1.03 health conditions technical personnel, and each bed shall be equipped with at least 0.4 nurses. The professional departments shall have the title of deputy chief physician or above. There shall be no less than 2 clinical nutritionists. The proportion of engineering and technical personnel (technician, assistant engineer or above) in the total number of health technical personnel shall not be less than $1 \%$

Technical level On the basis of high-quality comprehensive medical services, we should provide high-level specialized services. Undertake the task of diagnosis and treatment of critical and difficult diseases, and carry out two-way referral. There should be enough radiation capacity for medical services, and a certain proportion of discharged patients should come from areas or provinces other than the location of the hospital. Under the leadership of the health administrative department, we can cooperate with the first aid center to make emergency response quickly, undertake the emergency rescue task of disaster accidents, and accept a batch of patients for hospital first aid. Carry out mental health, outpatient services and support, guide community medical, nursing, rehabilitation medical services

Hospital tasks Regional or above hospitals that provide high-level and functions specialized medical and health services and carry out higher education and scientific research tasks in several regions

Teaching and To undertake clinical teaching and practice in Higher research Medical College, and to cultivate senior clinical medical talents. And undertake the clinical professional training task of technical backbone in secondary hospital. To undertake national, provincial (autonomous region, municipality) scientific research projects
Each bed shall be equipped with at least 0.7-0.88 health technical personnel, each bed shall be equipped with at least 0.4 nurses, at least 3 doctors with the title of deputy chief physician or above, and each professional department shall have at least 1 doctor with the title of attending physician or above

It can carry out the diagnosis and treatment of common and frequently occurring diseases in various departments of the community, and the rescue service for general critically ill patients

Primary hospitals and health centers providing prevention, medical treatment, health care and rehabilitation services to communities with a certain population. Or regional hospitals that provide comprehensive medical and health services to multiple communities and undertake certain teaching and scientific research tasks

It can undertake the further education and training of all kinds of health technical personnel in primary medical units and the on-the-job education of staff in the hospital. Be able to undertake the clinical teaching of secondary health school and the clinical practice task of medical school students above secondary health school. Able to undertake provincial or municipal scientific research projects 